\title{
Ahnak1 abnormally localizes in muscular dystrophies and contributes to muscle vesicle release
}

\author{
Ute Zacharias • Bettina Purfürst • Verena Schöwel • \\ Ingo Morano $\cdot$ Simone Spuler $\cdot$ Hannelore Haase
}

Received: 24 August 2011/Accepted: 19 October 2011/Published online: 5 November 2011

(C) The Author(s) 2011. This article is published with open access at Springerlink.com

\begin{abstract}
Ahnak1 is a giant, ubiquitously expressed, plasma membrane support protein whose function in skeletal muscle is largely unknown. Therefore, we investigated whether ahnak would be influenced by alterations of the sarcolemma exemplified by dysferlin mutations known to render the sarcolemma vulnerable or by mutations in calpain3, a protease known to cleave ahnak. Human muscle biopsy specimens obtained from patients with limb girdle muscular dystrophy (LGMD) caused by mutations in dysferlin (LGMD2B) and calpain3 (LGMD2A) were investigated for ahnak expression and localization. We found that ahnak1 has lost its sarcolemmal localization in LGMD2B but not in LGMD2A. Instead ahnak1 appeared in muscle connective tissue surrounding the extracellular site of the muscle fiber in both muscular dystrophies. The entire giant
\end{abstract}

This is a contribution to a Special Issue to EMC 2011.

Electronic supplementary material The online version of this article (doi:10.1007/s10974-011-9271-8) contains supplementary material, which is available to authorized users.

U. Zacharias · V. Schöwel $\cdot$ S. Spuler

Muscle Research Unit, Experimental and Clinical Research

Center, a joint cooperation between the Charité Medical

Faculty and the Max-Delbrück Center for Molecular Medicine,

Berlin, Germany

B. Purfürst · I. Morano $\cdot$ H. Haase $(\bowtie)$

Max Delbrück Center for Molecular Medicine, Muscle

Physiology Group, Robert-Rössle-Str. 10, 13125 Berlin,

Germany

e-mail: haase@mdc-berlin.de

I. Morano

University Medicine Charité, Berlin, Germany ahnak1 molecule was present outside the muscle fiber and did only partially colocalize with CD45-positive immune cell infiltration and the extracelluar matrix proteins fibronectin and collagenVI. Further, vesicles shedded in response to $\mathrm{Ca}^{2+}$ by primary human myotubes were purified and their protein content was analysed. Ahnak1 was prominently present in these vesicles. Electron microscopy revealed a homogenous population of vesicles with a diameter of about $150 \mathrm{~nm}$. This is the first study demonstrating vesicle release from human myotubes that may be one mechanism underlying abnormally localized ahnak1. Taken together, our results define ahnak1 in muscle connective tissue as a novel feature of two genetically distinct muscular dystrophies that might contribute to disease pathology.

Keywords Limb girdle muscular dystrophy - Ahnak . Dysferlin · Calpain3 - Endomysial connective tissue . Vesicle shedding

\section{Introduction}

Proper skeletal muscle function relies on muscle fiber integrity, efficient muscle repair and regeneration which are disturbed in muscular dystrophies (Goldstein and McNally 2010). Limb girdle muscular dystrophies (LGMD) comprise clinically and genetically heterogenous disorders characterized by progressive wasting of the skeletal muscle combined with an increase in muscle connective tissue. LGMD type 2B and LGMD type 2A are caused by mutations in dysferlin (MIM*603009) and calpain3 (MIM*114240), respectively (Bashir et al. 1998; Richard et al. 1995). Dysferlin is a sarcolemmal protein involved in plasma membrane repair and muscle regeneration (Bansal et al. 2003; Chiu et al. 2009). Mutations in dysferlin lead to reduced 
expression and/or altered subcellular localization of the protein in muscle fibers (Wenzel et al. 2006; Spuler et al. 2008; Krahn et al. 2009). Calpain 3 is a cystein protease that cleaves cytoskeletal and myofibrillar proteins and is reported to serve as gatekeeper of proper sarcomere function (Kramerova et al. 2005; Beckmann and Spencer 2008).

Ahnak1 (desmoyokin, MIM*103390) interacts with dysferlin and is cleaved by calpain 3 (Huang et al. 2007, 2008). Ahnak1 is a large protein implicated in plasma membrane support (Hohaus et al. 2002; Gentil et al. 2003) and $\mathrm{Ca}^{2+}$ signaling in a variety of cells (Hashimoto et al. 1995; Haase 2007; Matza et al. 2008; Alli and Gower 2009; Alvarez et al. 2010). Much attention has been paid to ahnak as a marker of enlargeosomes, which are non-secretory, cytoplasmic vesicles competent for regulated exocytosis after rising intracellular $\mathrm{Ca}^{2+}$. Enlargeosomes mediate cell surface enlargement and contribute to plasma membrane repair and vesicle shedding (Borgonovo et al. 2002; Cocucci et al. 2007; Lorusso et al. 2006). Little is known about ahnak function in skeletal muscle. Recently, ahnak1 and ahnak2 have been described as costameric proteins (Marg et al. 2010). Furthermore, ahnak has been identified as a constituent of the dysferlin protein complex that is important for sarcolemmal integrity (Huang et al. 2007, 2008; de Morree et al. 2010). Interestingly, the interaction between ahnak1 and dysferlin is lost after proteolytic processing of ahnak1 by calpain3 (Huang et al. 2008).

Because of the complex interactions between ahnak1, dysferlin, and calpain 3 and their role in muscle repair and regeneration we compared ahnak 1 expression in patients with LGMD2B and LGMD2A. We found an altered expression of ahnak1 at the sarcolemma and identified ahnak1 as a novel constituent of the endomysial compartment in the two genetically distinct muscular dystrophies studied. Furthermore, we demonstrate the release of ahnak1-containing vesicles from normal human myotubes after rising intracellular $\mathrm{Ca}^{2+}$. We infer that ahnak1 release by secreted vesicles may be one mechanism underlying abnormally localized ahnak1 in muscular dystrophy.

\section{Materials and methods}

\section{Patients}

The Charité internal review board approved the study and written informed consent was obtained from all patients. Skeletal muscle biopsies (M. vastus lateralis) from 6 patients with dysferlinopathy, 6 patients with calpainopathy and 6 healthy controls were analyzed. Patients with LGMD2B were affected by different mutations in DYSF including homozygous c.4022T $>\mathrm{C}$; homozygous c. $2810+2 \mathrm{~T}>$ $\mathrm{A} ;$ compound heterozygous c.855 $+1 \mathrm{delG}$ and c. $895 \mathrm{G}>\mathrm{A}$; compound heterozygous $\mathrm{c} .1448 \mathrm{C}>\mathrm{A}$ and c. ${ }^{*} 107 \mathrm{~T}>\mathrm{A}$; compound heterozygous $\mathrm{c} .5606 \mathrm{G}>\mathrm{A}$ and c. $2875 \mathrm{C}>\mathrm{T}$ (see Table 2 in (Wenzel et al. 2006)) resulting in the complete loss or intracellular accumulation of dysferlin, respectively. Patients with LGMD2A were affected by different compound heterozygous mutations in the calpain3 gene including c.550delA and c. $1745+5 \mathrm{G}>\mathrm{C}$; c.550delA and c.883_886delGATAinsCTT; c.1469G > A and c.1801-1G $>$ A; c.700G $>A$ and $\quad$ c.1324T $>$ C; c.551C $>\mathrm{T}$ and c. $801+1 \mathrm{G}$ resulting in the complete loss or reduced expression of calpain3, respectively.

Antibodies against ahnak

The isoform-specific ahnak1 antibody was prepared by immunizing rabbits with a $\mathrm{C}$-terminal recombinant ahnak 1 protein fragment (amino acid residues 4893-5535) as recently described (Marg et al. 2010). In some experiments ahnak was labeled with the KIS antibody that was raised against the internal repeating units of ahnak (KISMPDVDLHLKGPK). The KIS antibody has been characterized elsewhere (Haase et al. 2004) and corresponds to the antibody used by Huang et al. (2007, 2008). The monoclonal antibody (M01, clone 3G7) against the N-terminal part of ahnak1 was purchased from Abnova.

\section{SDS-PAGE and Western blot analysis}

Total protein fractions were extracted from frozen tissue specimens and myotubes by homogenization with SDSsample buffer as previously described (Haase et al. 2004). Exosomal proteins were dissolved in SDS-sample buffer and heated at $95^{\circ} \mathrm{C}$ for $5 \mathrm{~min}$. Protein samples were separated by SDS-PAGE and transferred to nitrocellulose filters overnight at $70 \mathrm{~mA}$ with a Tris/glycine buffer, $\mathrm{pH} 8.3$, containing $10 \%$ methanol and $0.1 \%$ SDS in a Biorad Protean III system. The transferred proteins were visualized by staining with Ponceau-S. Membranes were further processed for immunodetection according to standard protocols. Briefly, they were subsequently incubated with the affinity-purified, polyclonal antibody against ahnak1 $(1 \mu \mathrm{g} \mathrm{IgG/ml})$ or monoclonal antibodies against desmin (DAKO) and annexin2 (BD Laboratories) for $90 \mathrm{~min}$ and the respective secondary horseradish peroxidase-conjugated antibodies (Pierce, Rockford, USA). Immunoreactive protein bands were visualized by Chemiluminescent HRP Substrate (Millipore, USA) and Amersham Hyperfilm (GE Healthcare, Japan).

Myoblast purification and differentiation

Biopsies were taken from M.vastus lateralis. Primary myoblast cultures were isolated by protease digestion from fresh muscle biopsies and expanded at $37^{\circ} \mathrm{C}$ in humidified 
atmosphere with $5 \% \mathrm{CO}_{2}$ in skeletal muscle growth medium (PromoCell, Heidelberg, Germany) supplemented with $10 \%$ FCS, glutamine $(3 \mathrm{mM})$ and gentamycin $(40 \mu \mathrm{g} / \mathrm{ml})$ (Gibco, Paisely, UK). All cultures were enriched in myoblasts by immuno-magnetic cell sorting using anti-CD56/ NCAM antibody coated magnetic beads (Miltenyi Biotech, Bergisch Gladbach, Germany). Purity of the myoblast preparation was verified by staining with an anti-desmin antibody (DAKO) revealing more than 95\% desmin-positive cells. Differentiation of myoblasts into myotubes was initiated at approximately $90 \%$ confluence by switching to differentiation medium (DMEM, 2\% horse serum) followed by cultivation for 7 days.

\section{Immunocytochemistry}

Myoblasts were grown in 8 well chamber slides (Nunc, Langenselbold, Germany) and differentiated into myotubes, fixed for 10 min with $4 \%$ formaldehyde, permeabilized for 15 min with $0.2 \%$ Triton X100 and blocked with $1 \%$ BSA diluted in PBS. The following primary antibodies were used for immunostaining: mouse monoclonal antibodies to MyHCs (Novocastra, Newcastle upon Tyne, UK), dystrophin (Abcam), $\alpha$-actinin and vinculin (both from Sigma-Aldrich) and rabbit polyclonal antibodies to human ahnak1 ( $3 \mu \mathrm{g} \mathrm{IgG} / \mathrm{ml})$ in combination with secondary Alexa 488-conjugated anti-mouse IgG or Alexa 594-conjugated anti-rabbit IgG antibodies (Invitrogen), respectively. Nuclei were counterstained with Hoechst 33258. Images were collected with a Leica DMI6000 fluorescence microscope.

\section{Immunohistochemistry}

Immunofluorescenct staining was performed on transversal $5 \mu \mathrm{m}$ cryostat sections of skeletal muscle biopsies (M.vastus lateralis) of patients with dysferlinopathy, calpainopathy and healthy controls. After fixation with aceton, muscle sections were blocked with $10 \%$ goat serum in PBS and stained with rabbit polyclonal anti-ahnak1, anti-fibronectin (Abcam) or KIS antibodies in combination with Alexa 594-conjugated anti-rabbit IgG antibodies (Invitrogen). Sections were double-labelled with a monoclonal antibody to dystrophin C-terminus (Novocastra), CD45 (BD Laboratories) or ahnak1-NT (Abnova) in combination with Alexa 488-conjugated anti-mouse IgG antibodies (Invitrogen) to highlight the sarcolemma. Negative control samples without primary or secondary antibodies were included and produced negligible fluorescence. Images were collected with a Leica DM6000 or BioRad MRC1024 microscope. To block specific interactions, rabbit polyclonal anti-ahnak1 antibody was preincubated with a 20-fold molar excess of the recombinant ahnak1-GST fusion protein used for immunization (see Antibodies) overnight at $4^{\circ} \mathrm{C}$ and applied to cryosections as described above. For semiquantitative analysis ahnak1 localization was grouped into four categories after visual inspection of sections from patients with calpainopathy, dysferlinopathy and healthy controls: absent, partially reduced or present at the sarcolemma and localized to the endomysium.

Vesicle purification and characterization

Secreted vesicles were isolated by differential centrifugation from cell culture supernatants of human myotubes differentiated in $10 \mathrm{~cm}$ diameter petri dishes. After $24 \mathrm{~h}$ in serum free media the myotubes were washed with fresh medium and then exposed, for $10 \mathrm{~min}$ at room temperature, to ionomycin $(1 \mu \mathrm{M}, 3.7 \mu \mathrm{M})$, phorbol(12-myristoyl-13-acetyl) $(0.5 \mu \mathrm{M})$, and ATP $(1 \mathrm{mM})$ or to their solvents, diluted in $2 \mathrm{ml}$ medium. At the end of the incubations, the media were harvested and subjected to differential centrifugation at $4^{\circ} \mathrm{C}$ starting with $4.000 \times g$ to remove large cell remnants. The resulting supernatants were filtered through a syringe filter with $0.22 \mu \mathrm{m}$ cut-off (Millipore), and the filtrates were centrifuged at $100.000 \times g$ in an Optima ultracentrifuge (Beckman-Coulter) for $2 \mathrm{~h}$ to pellet vesicles which were only visible upon ionomycin treatment. The pellets ( $\sim 50 \mu \mathrm{g}$ of protein/petri dish) were either resuspended for Western blotting or were processed for negative staining and electron microscopy. For the latter method, the preparations were fixed in $2 \%$ freshly prepared formaldehyde in $200 \mathrm{mM}$ HEPES buffer, pH 7.4 over night. Small aliquots of this mixture were applied to formvar-carbon-coated grids for $5 \mathrm{~min}$, washed shortly with bidistilled water and stained with $5 \%$ aqueous uranyl acetate. Images were taken with a Zeiss 910 electron microscope equipped with a $1 \mathrm{k} \times 1 \mathrm{k}$ high speed slow scan CCD camera and the iTEM software (Olympus Soft Imaging Solutions, Münster, Germany).

\section{Results}

Ahnak1 is mislocalized in dysferlinopathy and calpainopathy

To examine potential alterations in the distribution of ahnak1 in muscular dystrophies caused by mutations in dysferlin and calpain3, we employed a recently characterized ahnak1 isoform-specific antibody (Marg et al. 2010). Cross sections of normal human skeletal muscle revealed a sarcolemma associated staining pattern for ahnak1 (Fig. 1, upper panel, green) that closely merged with dystrophin labeling (Fig. 1, upper panel, red). Next we analyzed the distribution of ahnak1 in patients with dysferlinopathy and calpainopathy. In muscle from a LGMD2B patient, plasma 

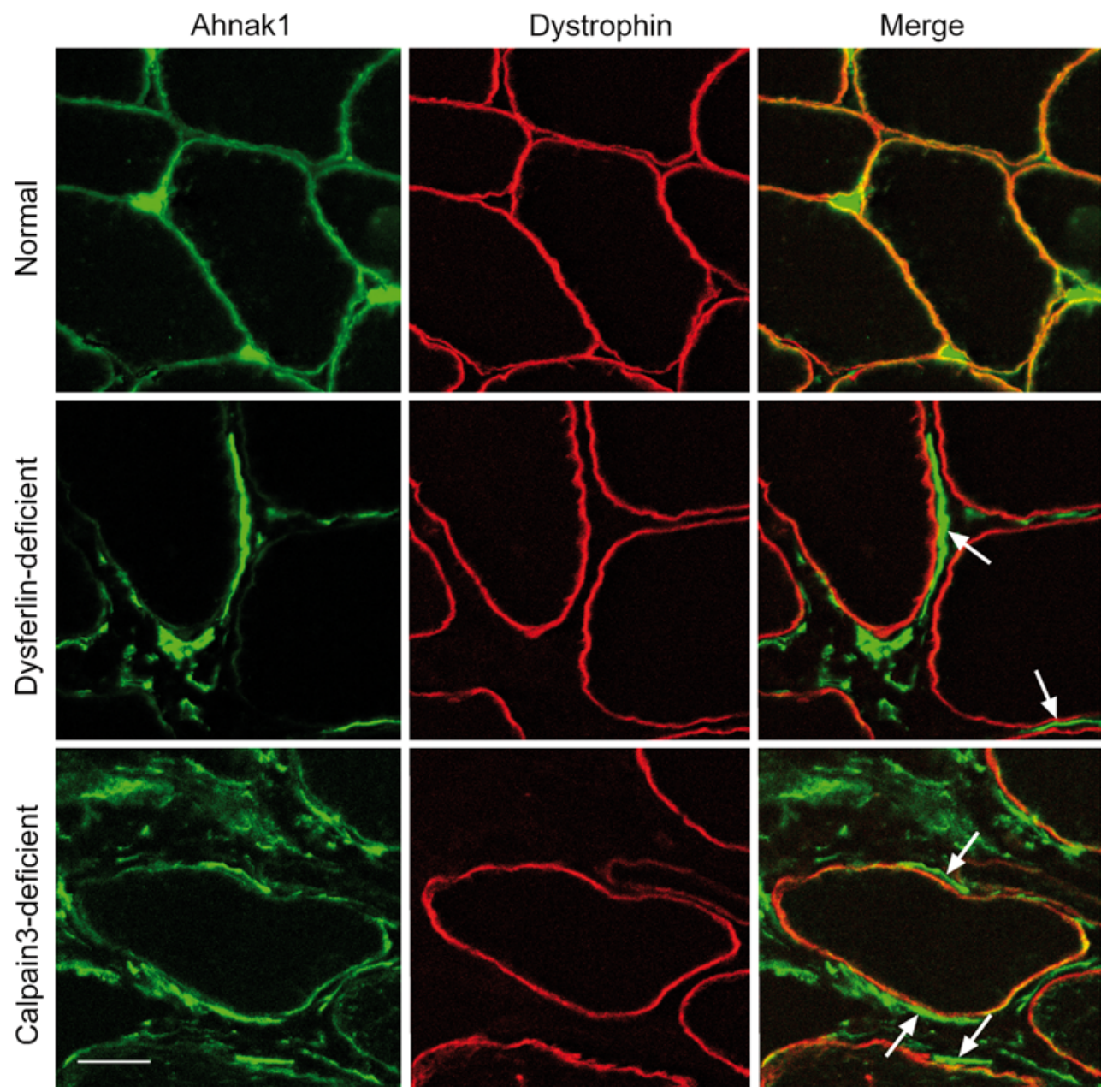

Fig. 1 Endomysial ahnak1 deposits surrounding the sarcolemma in dysferlinopathy and calpainopathy. Cross-sections of human skeletal muscle were stained for ahnak1 together with dystrophin that highlights the sarcolemma. Confocal microscopy of normal human skeletal muscle demonstrated sarcolemmal localization of ahnak1 (green), together with dystrophin (red; upper panel). Skeletal muscle

membrane staining with antibodies to dystrophin revealed myopathic changes typical for dystrophic muscle, such as pathological variations in fiber size, split fibers, and an increase in endomysial fibrosis (Fig. 1, middle panel, red). Ahnak1 labeling at the sarcolemma was dramatically reduced in all six patients with dysferlinopathy, compared to normal skeletal muscle. We show a representative example in Fig. 1 (middle panel, green). Remarkably, we observed strong immunostaining for ahnak1 outside the muscle fibers in the endomysium and perimysium in dysferlinopathy (arrows in Fig. 1, middle panel). Sections of skeletal muscle from patients with calpainopathy demonstrated strong dystrophic changes including increased endomysial fibrosis, as revealed by sarcolemmal membrane staining for dystrophin (Fig. 1, lower panel, red). In all six patients with calpain 3 mutations, ahnak1 was unchanged or only slightly reduced at the sarcolemma (Fig. 1, lower from an exemplary patient with dysferlinopathy (middle panel) and an exemplary patient with calpainopathy (lower panel) revealed strong dystrophic changes combined with increased endomysial localization of ahnak1. Ahnak1 formed line structures outside the muscle fibers, often parallel to the plasma membrane (arrows; bar, $25 \mu \mathrm{m}$ )

panel, green). Interestingly, merged images revealed strong ahnak1 labeling outside the muscle fiber within the endomysium in calpainopathy (arrows in Fig. 1, lower panel) very similar to dysferlin-deficient muscle as described above. Thus, in both these dystrophies, ahnak1 was present outside the muscle fiber in the endomysium and formed line structures partially ensheathing the muscle fiber (arrows in Fig. 1, middle and lower panel). Ahnak1 localization in patients and healthy controls is summarized in Online Resource 1. The specificity of ahnak1 immunostaining in normal and dystrophic muscle was verified by blocking ahnak1 labeling with the recombinant ahnak1 protein used to generate the antibody (Online Resource 2a).

We next addressed the question whether ahnak1 fragments or the full-length protein reside outside the muscle fiber in the endomysial compartment. Double labeling of ahnak1 with antibodies directed against either the 


\section{a}
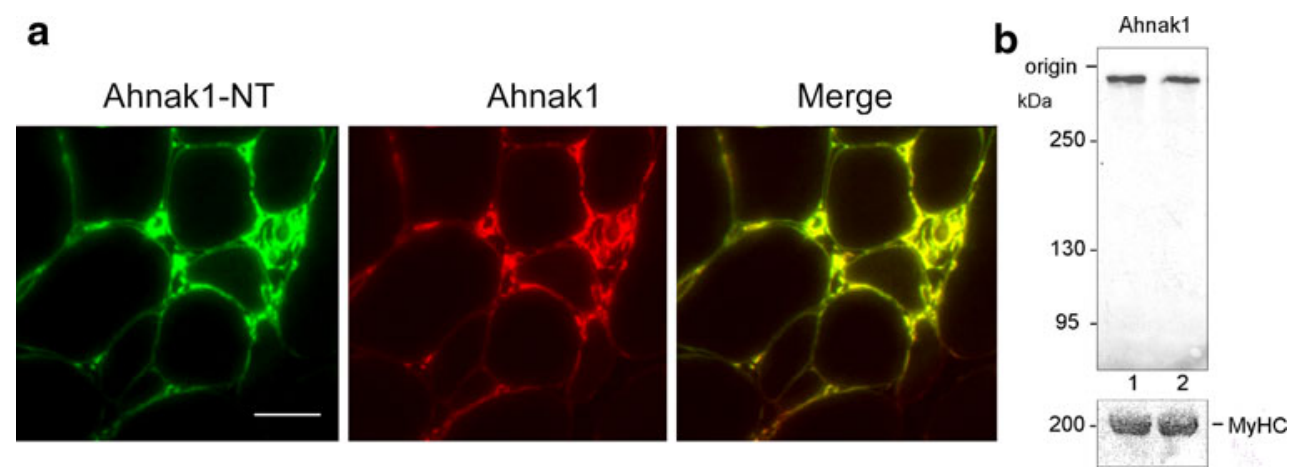

Fig. 2 Ahnak1 expression analyzed with domain-specific antibodies in dystrophic muscle. a Ahnak1 labeling in a calpainopathy patient with anti ahnak1-NT mAb and anti ahnak1 pAb directed against the $\mathrm{N}$-terminal and $\mathrm{C}$-terminal region of ahnak1, respectively. There was a strong co-localization of the $\mathrm{N}$-terminal and C-terminal parts of

$\mathrm{N}$-terminus or the C-terminus of the $700-\mathrm{kDa}$ protein revealed perfect colocalization for both ahnak1 epitopes in calpain3-deficient muscle (Fig. 2a) and dysferlin-deficient muscle (data not shown). Western blots of tissue homogenates from normal and dystrophic human skeletal muscle revealed a single immunoreactive band for ahnak 1 with the expected high molecular mass (Fig. 2b). These findings suggest that the entire giant ahnak 1 molecule is present in the enlarged endomysium in both forms of muscular dystrophies.

Furthermore, we used the KIS antibody for ahnak labeling in muscle sections. This antibody was generated against the repeating units of ahnak 1 and is expected to detect both ahnak 1 and ahnak2. We observed strong, endomysial ahnak1/2 labeling in dysferlin-deficient and calpain3-deficient patients (Online Resource 2b) that is consistent with a localization of ahnak outside the muscle fiber as shown above with the ahnak1 isoform-specific antibody.

Extracellular ahnak1 in relation to immune infiltration and interstitial fibrosis

To shed light on the origin of endomysial ahnak1, we considered the possibility that ahnak1 was derived from cells invading the endomysial space such as activated macrophages and lymphocytes, in which ahnak1 expression is well documented (Matza et al. 2008; Matza and Flavell 2009). We thus combined ahnak1 labeling with staining for CD45, a marker for immune cells (Fig. 3). In healthy human skeletal muscle, infiltration of immune cells was hardly visible. Only a single rounded cell was detected outside the skeletal muscle fiber that was labeled for both CD45 and ahnak1 (Fig. 3, upper panel). Dystrophic muscle from dysferlin-deficient and calpain3-deficient patients revealed significant CD45-positive immune cell infiltration that partially co-localized with ahnak1 labeling. However, ahnak1 showed a broader distribution and ahnak1-positive ahnak1 (bar, $50 \mu \mathrm{m})$. b Western blots from a healthy control (lane 1) and from a patient with calpainopathy (lane 2) showed ahnak1 migrating as a single $\sim 700 \mathrm{kDa}$ protein in both samples. Protein staining of MyHC was used as loading control

line structures ensheathing the muscle fibers were not labeled by CD45 (Fig. 3, middle and lower panel). Thus, infiltration of immune cells was probably not the only reason for the presence of ahnak1 in the endomysium.

To relate ahnak1 expression to interstitial fibrosis in dystrophic muscle, we compared its localization with fibrosis markers, namely the extracellular matrix (ECM) proteins fibronectin and collagen VI (Alvarez et al. 2002; Fadic et al. 2006). In healthy specimen, fibronectin was only slightly expressed and was preferentially localized at blood vessels between muscle fibers and in the perimysial space (Fig. 4, upper panel). The increase in fibrosis in dystrophic muscle was accompanied by an increase in fibronectin expression in the endomysium in both dysferlinopathy and calpainopathy (Fig. 4, middle and lower panel, respectively). However, fibronectin and ahnak1 showed only a partially overlapping expression pattern in the endomysium in dystrophic muscle. Fibronectin distribution was broader and again the characteristic ahnak1-positive structures embracing the sarcolemma were not highlighted by fibronectin (Fig. 4, middle and lower panel). Moreover, in dystrophic muscle ahnak1 showed a localization distinct from the ECM molecule, collagen VI, and the basal lamina marker, laminin $\alpha 2$ (Fig. 5). These findings support the presence of ahnak1 outside the muscle fiber in dystrophic muscle. However, ahnak1 does not entirely co-localize with the ECM proteins fibronectin, collagen VI, and laminin $\alpha 2$.

\section{May extracellular ahnak1 originate from muscle?}

We tested the hypothesis whether ahnak outside the muscle fiber may be released from muscle through secreted vesicles. We employed primary human myotubes as a model and adapted protocols for vesicle shedding (Cocucci et al. 2007; Lorusso et al. 2006). Multinucleated myotubes were formed from normal primary human myoblasts after 7 days 

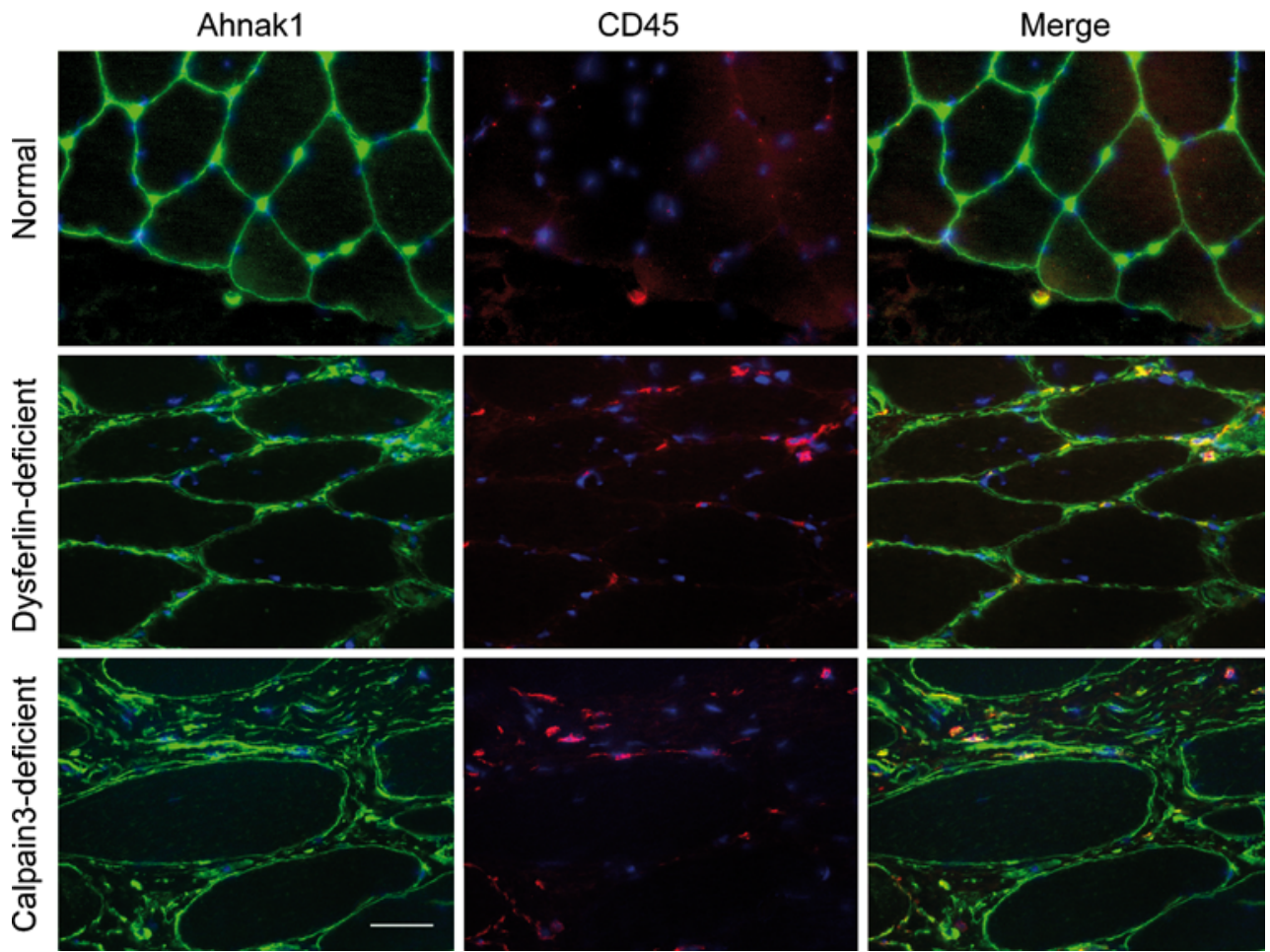

Fig. 3 Ahnak1 expression and infiltration of immune cells in dystrophic human skeletal muscle. Cross-sections of normal human skeletal muscle (upper panel), a patient with dysferlinopathy (middle panel) and a patient with calpainopathy (lower panel) were labeled for ahnak1 (green) and CD45 (red) as an immune cell marker (bar, $50 \mu \mathrm{m})$
Fig. 4 Comparison of ahnak1 and fibronectin expression in normal and dystrophic human skeletal muscle. Cross-sections of normal human skeletal muscle (upper panel), an exemplary patient with dysferlinopathy (middle panel), and a patient with calpainopathy (lower panel) were labeled for ahnak1 (anti ahnak1-NT mAb; green) and fibronectin (red) as a marker for endomysial fibrosis (bar, $50 \mu \mathrm{m}$ )
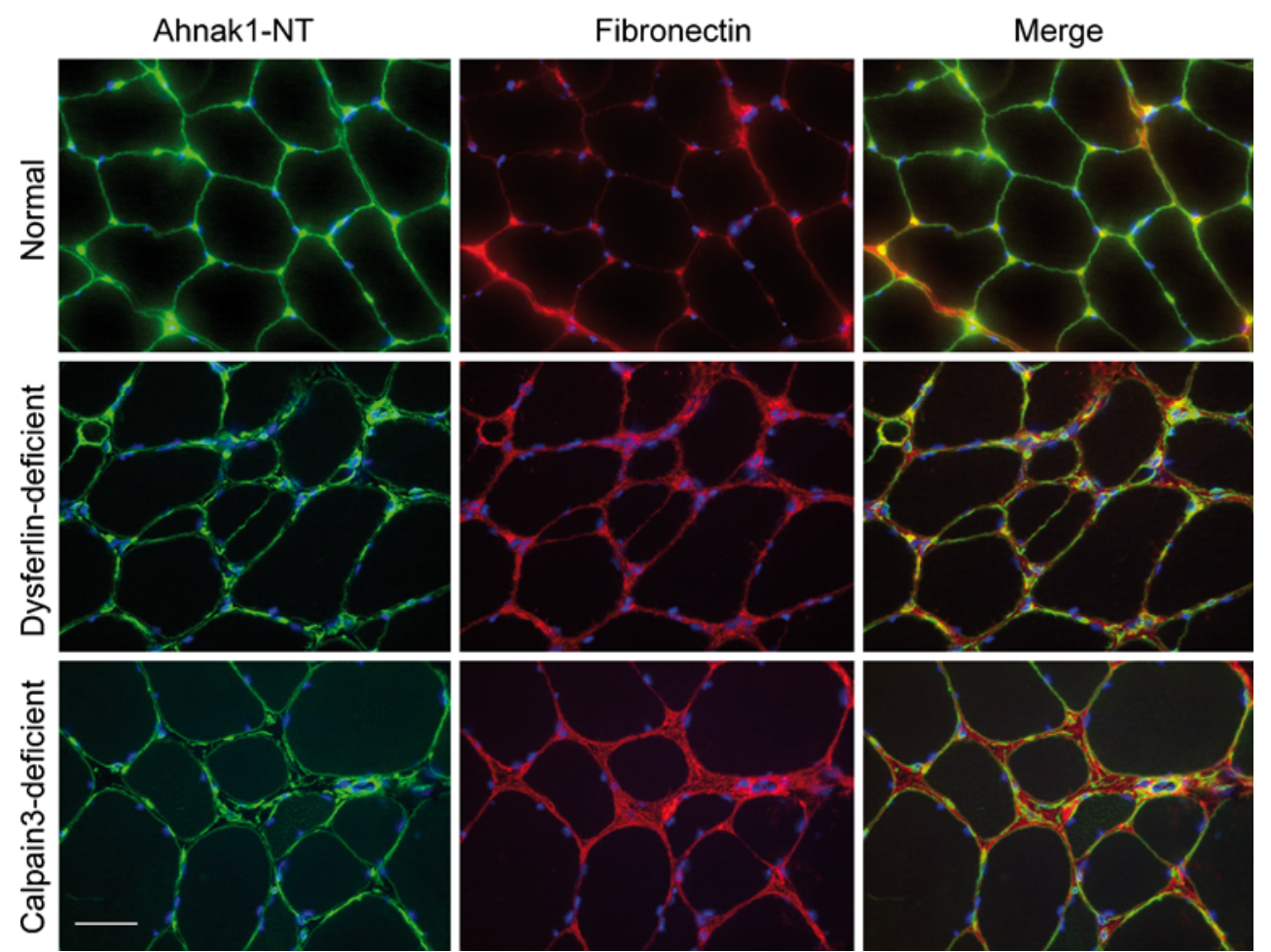

of differentiation. At this state of maturation the myotubes showed a striated staining pattern for myosin heavy chain (MyHC) and $\alpha$-actinin (labels Z-lines) indicative for the formation of sarcomeres (Fig. 6). Costamere-like structures were not yet developed. The costameric proteins vinculin and dystrophin showed a focal adhesion-like staining 
a

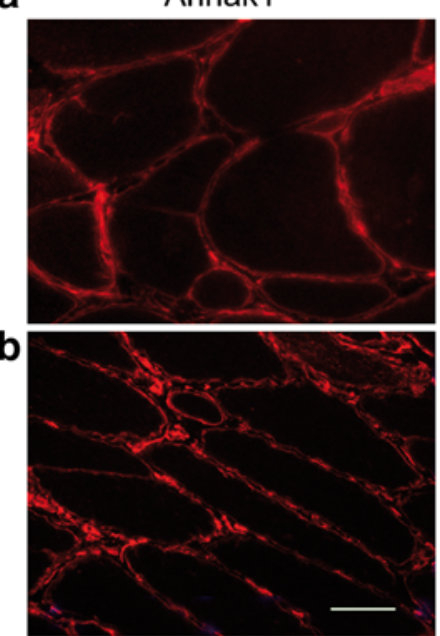

Ahnak1

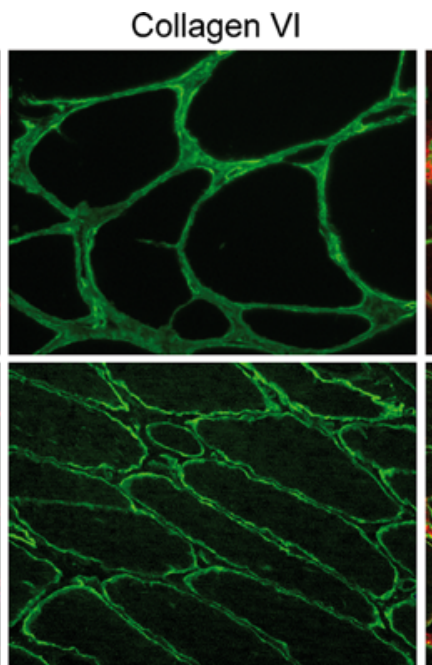

Laminin $\alpha 2$

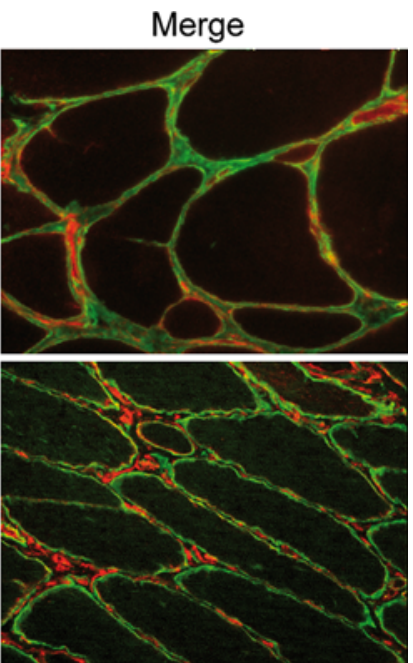

Merge
Fig. 5 Comparison of collagen VI, laminin $\alpha 2$ and ahnak1 expression in dystrophic human skeletal muscle. Cross-sections of human skeletal muscle of an exemplary patient with calpainopathy (a) and

pattern and an intracellular localization, respectively (Fig. 6). In myotubes ahnak1 was distributed throughout the cytoplasm and at the plasma membrane (Fig. 6). Ahnak1 staining was only observed after permeabilization of cells indicating that ahnak1 is not exposed at the outer surface of the plasma membrane (data not shown).

To evoke vesicle shedding we incubated cultured adherent myotubes with PMA, ionomycin, ATP or vehicle as control. Media were harvested after $10 \mathrm{~min}$ of drug application and were subjected to differential centrifugation as outlined in Materials and methods. The high speed pellet following $100.000 \times g$ centrifugation was considered as secreted vesicles and their protein composition was analysed by Western blotting. Figure 7a clearly demonstrates a patient with dysferlinopathy (b) were labeled for ahnak1 (red) and collagen VI or laminin $\alpha 2$ (green), as a marker for endomysial fibrosis and basal lamina, respectively (bar, $50 \mu \mathrm{m})$

that this fraction contained ahnak1 together with annexin 2 upon treatment with the $\mathrm{Ca}^{2+}$ ionophore ionomycin, whereas the intermediate filament protein desmin was absent. Robust release of ahnak1 into the vesicular fraction was repeatedly seen at a concentration of $1 \mu \mathrm{M}$ ionomycin, while PMA $(0.5 \mu \mathrm{M}$, Fig. 7a) and ATP (1 $\mu \mathrm{M}$, data not shown) failed to induce the release of any constituent into the high speed pellet. Electron microscopy was performed to characterize the vesicular fraction released by ionomycin treatment. Representative images depicted in Fig. 7b revealed that this myotube-derived preparation contained rounded vesicles with a diameter of 150-200 nm. The characteristics of repeated vesicle preparations are given in Table 1. It is noteworthy to mention that an additional

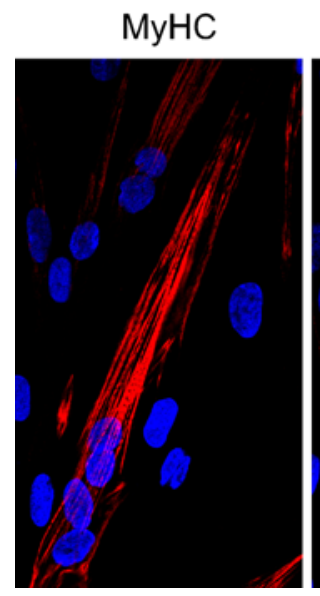

$\alpha$ Actinin

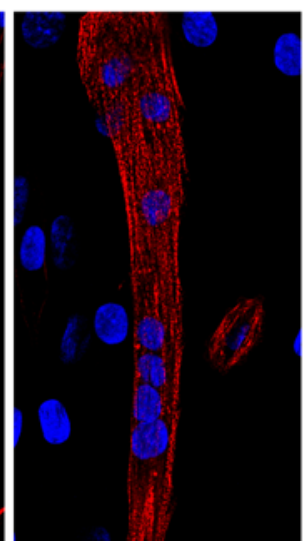

Vinculin

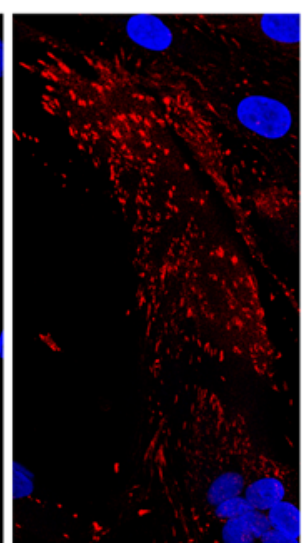

Dystrophin

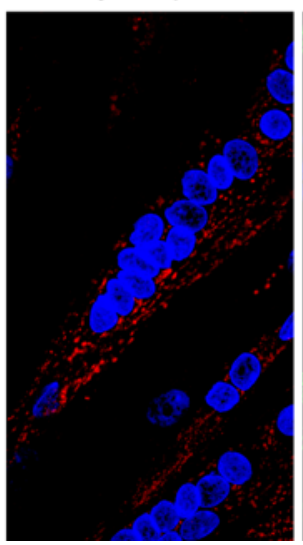

Ahnak1

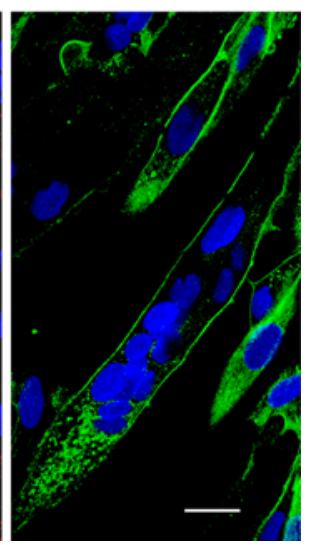

Fig. 6 Differentiation state of primary human myotubes. Immunofluorescence staining with either anti-MyHC or with anti- $\alpha$ actinin antibody showed correct organization of the sarcomere. Costamere- like structures were not yet developed, as shown by staining with antivinculin or anti-dystrophin antibody. Ahnak1 was distributed throughout the cytoplasm and at the plasma membrane (bar, $20 \mu \mathrm{m})$ 

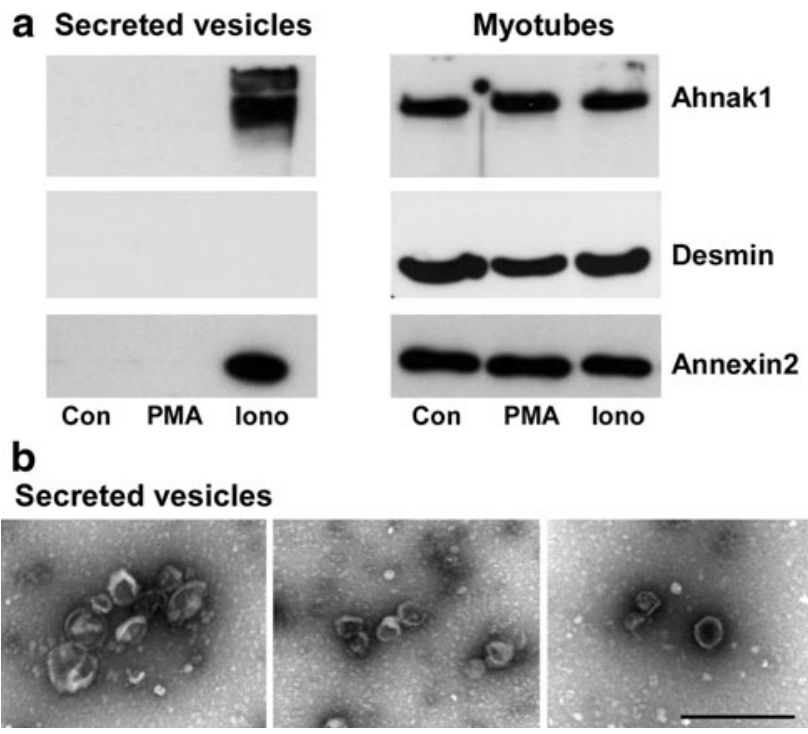

Fig. 7 Ahnak1 and annexin2 are present in vesicles secreted from human myotubes. a Myotubes were incubated with $0.5 \mu \mathrm{M}$ PMA, $1 \mu \mathrm{M}$ ionomycin (Iono), or vehicle as control (Con) for $10 \mathrm{~min}$. Ahnak1 and annexin2 are present in vesicles secreted by ionomycin treatment (left panel). The corresponding myotubes express ahnak1, annexin2 and desmin (right panel) as assessed by Western blotting. b Electron micrographs of secreted vesicles purified from ionomycin treated myotubes. Representative images show rounded vesicles of $150-200 \mathrm{~nm}$ in diameter (bar, $500 \mathrm{~nm}$ )

Table 1 Size distribution of vesicles secreted from human myotubes

\begin{tabular}{lllll}
\hline Preparation & Filter & $\begin{array}{l}\text { Vesicles } \\
\text { counted }\end{array}$ & $\begin{array}{l}\text { Mean } \\
\text { diameter }(\mathrm{nm})\end{array}$ & $\begin{array}{l}\text { Standard } \\
\text { deviation }(\mathrm{nm})\end{array}$ \\
\hline 1 & No & 119 & 178.6 & 56.7 \\
2 & No & 206 & 211.8 & 60.4 \\
3 & Yes & 154 & 147.9 & 54.8 \\
4 & Yes & 185 & 154.2 & 54.9 \\
\hline
\end{tabular}

Vesicles were released from human myotubes by ionomycin treatment $(1 \mu \mathrm{M}, 10 \mathrm{~min})$, separated by differential centrifugation and analyzed by electron microscopy. Size distribution did not differ with or without filtration through a $0.22 \mu \mathrm{m}$ filter

purification step through a $0.22 \mu \mathrm{m}$ filter did essentially not alter the dimensions of secreted vesicles. Importantly, no evidence of contamination with other membrane particles or cell remnants was observed in our vesicle preparation (Fig. 7b).

\section{Discussion}

The important finding in our study is the localization of ahnak1 outside the muscle fiber in patients with calpainopathy (LGMD2A) and dysferlinopathy (LGMD2B). We believe this information implies that ahnak1 is faultily regulated in the muscular dystrophies we investigated.
Elucidation of the mechanisms involved in ahnak1 mislocalization could help explain these muscle diseases further.

Healthy human skeletal muscle did not show endomysial ahnak1. In cross sections, we observed a densely packed, plasma membrane associated staining pattern for ahnak 1 that closely merged with dystrophin labeling. Plasma membrane localization is in line with previous reports and supports the functional role of ahnak in skeletal muscle as a plasma membrane stabilizer and costamer-associated protein (Hohaus et al. 2002; Haase et al. 2004; Gentil et al. 2003; Benaud et al. 2004; Marg et al. 2010). Specifically, in patients with LGMD2B a separation between dystrophin and ahnak1 became apparent as ahnak1 labeling at the muscle membrane was strongly reduced. This finding is in agreement with Huang et al. (2007) who described a concerted loss of dysferlin and ahnak at the plasma membrane in dysferlinopathy patients. Loss of ahnak from the sarcolemma seems to be characteristic for dysferlinopathies because it is not observed in calpainopathies. However, endomysial localization of ahnak1 appeared to be a common feature in both muscle diseases. In fact, we found ahnak1 accumulation in the enlarged interstitial compartment of dysferlin-deficient and calpain3-deficient muscle. Again, co-immunostaining of ahnak1 and dystrophin allowed clear differentiation between plasma membrane labeling and endomysial labeling. Ahnak1 staining alone could have been misinterpreted as sarcolemmal thickening or multilayered basal lamina. Several antibodies against different domains of ahnak1 including $\mathrm{N}$ - and $\mathrm{C}$-terminus as well as the repeating units support ahnak1 deposition outside the dystrophic muscle fiber. Furthermore, we corroborated the localization of ahnak1 outside the muscle fibers in the two diseases by partial co-labeling with the extracellular matrix molecules fibronectin and collagen VI. These proteins are accepted constituents of endomysial connective tissue in the dystrophic muscle.

In general, muscular dystrophies are characterized by a progressive muscle weakness and wasting, in combination with fibrosis that contributes to disease severity (Alvarez et al. 2002; Fadic et al. 2006; Goldstein and McNally 2010). Excessive accumulation of matrix components in the endomysium may have strong effects on the activation and differentiation of resident cells, and therefore muscle regeneration. Skeletal muscle fibers rely on a proper connection with matrix to transduce muscle tension and to maintain cell integrity. Endomysial deposition of ahnak1 could influence these processes. A barrier function for ahnak expressing cells has been described after spinal cord injury and at the blood brain barrier (Salim et al. 2009; Gentil et al. 2005; von Boxberg et al. 2006). A similar effect might be mediated by the ahnak1 positive line structures partially ensheathing dystrophic muscle fibers. Therefore we suggest that ahnak1 deposition outside the 
muscle fiber may contribute to impaired muscle function in LGMD patients.

An interesting question arising from the present study is the localization of ahnak1 in other muscular dystrophies. We studied patients with Becker muscular dystrophy but our material was limited to two muscle biopsy specimens. Our preliminary data reveal aberrant ahnak1 localization in the endomysium in these two patients. This may indicate that extracellular ahnak1 might be a more general phenomenon in muscular dystrophies and is a subject of our ongoing research.

Another important finding of our study is the identification of ahnak1 in small vesicles $(\sim 150 \mathrm{~nm})$ secreted from myotubes after treatment with ionomycin to induce $\mathrm{Ca}^{2+}$ rise. This resembles the process of $\mathrm{Ca}^{2+}$ induced release of enlargeosomes which are non-secretory, cytoplasmic organelles competent for regulated exocytosis. Enlargeosomes participate in plasma membrane enlargement and repair and contain ahnak1 and annexin 2 as marker proteins (Borgonovo et al. 2002). Ahnak1-rich membranes derived from enlargeosome exocytosis undergo either endocytosis or release in shedding vesicles (Cocucci et al. 2007, 2009; Lorusso et al. 2006). In general, vesicle release into the extracellular environment represents a way for discarding proteins and membrane but also for intercellular communication through the transfer of proteins, RNA, and miRNA (Cocucci et al. 2009). Shedded vesicles can also serve as diagnostic markers. Very recently, ahnak1-containing vesicles have been detected in the sera of patients with pancreatic cancer (Uozumi et al. 2010). To our knowledge here we demonstrate for the first time the release of ahnak1-containing vesicles from differentiated human myotubes. Thus, endomysial ahnak1 may originate from skeletal muscle cells in muscular dystrophies following repeated cycles of degeneration and regeneration. However, other sources of endomysial ahnak1 remain to be investigated. We can not exclude that it originates from other cell types like fibroblasts, immune, endothelial, or smooth muscle cells or could simply represent cellular remnants.

In summary, the pivotal finding in our study is the identification of ahnak 1 as novel constituent of endomysial connective tissue in patients with calpainopathy (LGMD2A) and dysferlinopathy (LGMD2B), two genetically distinct muscular dystrophies. We demonstrate for the first time the release of ahnak1-containing vesicles from myotubes that may be one mechanism underlying ahnak1 mislocalization. The perspectives of our study lie in unravelling the mechanisms of ahnak1 displacement and its contribution to impaired muscle function.

Acknowledgments The Deutsche Forschungsgemeinschaft funded the study through the clinical research group KRO192/1 "Skeletal muscle growth regulation and dysregulation". We are grateful to Prof. Friedrich Luft for critical reading of the manuscript. We thank Stephanie Meyer, Steffen Lutter, and Karin Karczewski for expert technical assistance.

Open Access This article is distributed under the terms of the Creative Commons Attribution Noncommercial License which permits any noncommercial use, distribution, and reproduction in any medium, provided the original author(s) and source are credited.

\section{References}

Alli AA, Gower WR Jr (2009) The C type natriuretic peptide receptor tethers AHNAK1 at the plasma membrane to potentiate arachidonic acid-induced calcium mobilization. Am J Physiol Cell Physiol 297(5):C1157-C1167

Alvarez K, Fadic R, Brandan E (2002) Augmented synthesis and differential localization of heparan sulfate proteoglycans in Duchenne muscular dystrophy. J Cell Biochem 85(4):703-713

Alvarez JL, Petzhold D, Pankonien I, Behlke J, Kouno M, Vassort G, Morano I, Haase H (2010) Ahnak1 modulates L-type $\mathrm{Ca}(2+)$ channel inactivation of rodent cardiomyocytes. Pflugers Arch 460(4):719-730

Bansal D, Miyake K, Vogel SS, Groh S, Chen CC, Williamson R, McNeil PL, Campbell KP (2003) Defective membrane repair in dysferlin-deficient muscular dystrophy. Nature 423(6936): $168-172$

Bashir R, Britton S, Strachan T, Keers S, Vafiadaki E, Lako M, Richard I, Marchand S, Bourg N, Argov Z, Sadeh M, Mahjneh I, Marconi G, Passos-Bueno MR, Moreira Ede S, Zatz M, Beckmann JS, Bushby K (1998) A gene related to Caenorhabditis elegans spermatogenesis factor fer- 1 is mutated in limbgirdle muscular dystrophy type 2B. Nat Genet 20(1):37-42

Beckmann JS, Spencer M (2008) Calpain 3, the "gatekeeper" of proper sarcomere assembly, turnover and maintenance. Neuromuscul Disord 18(12):913-921

Benaud C, Gentil BJ, Assard N, Court M, Garin J, Delphin C, Baudier $\mathrm{J}$ (2004) AHNAK interaction with the annexin 2/S100A10 complex regulates cell membrane cytoarchitecture. J Cell Biol 164(1):133-144

Borgonovo B, Cocucci E, Racchetti G, Podini P, Bachi A, Meldolesi J (2002) Regulated exocytosis: a novel, widely expressed system. Nat Cell Biol 4(12):955-962

Chiu YH, Hornsey MA, Klinge L, Jorgensen LH, Laval SH, Charlton R, Barresi R, Straub V, Lochmuller H, Bushby K (2009) Attenuated muscle regeneration is a key factor in dysferlindeficient muscular dystrophy. Hum Mol Genet 18(11): 1976-1989

Cocucci E, Racchetti G, Podini P, Meldolesi J (2007) Enlargeosome traffic: exocytosis triggered by various signals is followed by endocytosis, membrane shedding or both. Traffic 8(6):742-757

Cocucci E, Racchetti G, Meldolesi J (2009) Shedding microvesicles: artefacts no more. Trends Cell Biol 19(2):43-51

de Morree A, Hensbergen PJ, van Haagen HH, Dragan I, Deelder AM, t Hoen PA, Frants RR, van der Maarel SM (2010) Proteomic analysis of the dysferlin protein complex unveils its importance for sarcolemmal maintenance and integrity. PLoS One 5(11):e13854. doi:10.1371/journal.pone.0013854

Fadic R, Mezzano V, Alvarez K, Cabrera D, Holmgren J, Brandan E (2006) Increase in decorin and biglycan in Duchenne Muscular Dystrophy: role of fibroblasts as cell source of these proteoglycans in the disease. J Cell Mol Med 10(3):758-769 
Gentil BJ, Delphin C, Benaud C, Baudier J (2003) Expression of the giant protein AHNAK (desmoyokin) in muscle and lining epithelial cells. J Histochem Cytochem 51(3):339-348

Gentil BJ, Benaud C, Delphin C, Remy C, Berezowski V, Cecchelli R, Feraud O, Vittet D, Baudier J (2005) Specific AHNAK expression in brain endothelial cells with barrier properties. J Cell Physiol 203(2):362-371

Goldstein JA, McNally EM (2010) Mechanisms of muscle weakness in muscular dystrophy. J Gen Physiol 136(1):29-34

Haase H (2007) Ahnak, a new player in beta-adrenergic regulation of the cardiac L-type $\mathrm{Ca}(2+)$ channel. Cardiovasc Res 73(1):19-25

Haase H, Pagel I, Khalina Y, Zacharzowsky U, Person V, Lutsch G, Petzhold D, Kott M, Schaper J, Morano I (2004) The carboxylterminal ahnak domain induces actin bundling and stabilizes muscle contraction. FASEB J 18(7):839-841

Hashimoto T, Gamou S, Shimizu N, Kitajima Y, Nishikawa T (1995) Regulation of translocation of the desmoyokin/AHNAK protein to the plasma membrane in keratinocytes by protein kinase $\mathrm{C}$. Exp Cell Res 217(2):258-266

Hohaus A, Person V, Behlke J, Schaper J, Morano I, Haase H (2002) The carboxyl-terminal region of ahnak provides a link between cardiac L-type $\mathrm{Ca} 2+$ channels and the actin-based cytoskeleton. Faseb J 16(10):1205-1216

Huang Y, Laval SH, van Remoortere A, Baudier J, Benaud C, Anderson LV, Straub V, Deelder A, Frants RR, den Dunnen JT, Bushby K, van der Maarel SM (2007) AHNAK, a novel component of the dysferlin protein complex, redistributes to the cytoplasm with dysferlin during skeletal muscle regeneration. Faseb J 21(3):732-742

Huang Y, de Morree A, van Remoortere A, Bushby K, Frants RR, Dunnen JT, van der Maarel SM (2008) Calpain 3 is a modulator of the dysferlin protein complex in skeletal muscle. Hum Mol Genet 17(12):1855-1866

Krahn M, Beroud C, Labelle V, Nguyen K, Bernard R, Bassez G, Figarella-Branger D, Fernandez C, Bouvenot J, Richard I, Ollagnon-Roman E, Bevilacqua JA, Salvo E, Attarian S, Chapon F, Pellissier JF, Pouget J, Hammouda el H, Laforet P, Urtizberea JA, Eymard B, Leturcq F, Levy N (2009) Analysis of the DYSF mutational spectrum in a large cohort of patients. Hum Mutat 30(2):E345-E375

Kramerova I, Kudryashova E, Venkatraman G, Spencer MJ (2005) Calpain 3 participates in sarcomere remodeling by acting upstream of the ubiquitin-proteasome pathway. Hum Mol Genet 14(15):2125-2134
Lorusso A, Covino C, Priori G, Bachi A, Meldolesi J, Chieregatti E (2006) Annexin2 coating the surface of enlargeosomes is needed for their regulated exocytosis. EMBO J 25(23):5443-5456

Marg A, Haase H, Neumann T, Kouno M, Morano I (2010) AHNAK1 and AHNAK2 are costameric proteins: AHNAK1 affects transverse skeletal muscle fiber stiffness. Biochem Biophys Res Commun 401(1):143-148

Matza D, Flavell RA (2009) Roles of Ca(v) channels and AHNAK1 in $T$ cells: the beauty and the beast. Immunol Rev 231(1): 257-264

Matza D, Badou A, Kobayashi KS, Goldsmith-Pestana K, Masuda Y, Komuro A, McMahon-Pratt D, Marchesi VT, Flavell RA (2008) A scaffold protein, AHNAK1, is required for calcium signaling during T cell activation. Immunity 28(1):64-74

Richard I, Broux O, Allamand V, Fougerousse F, Chiannilkulchai N, Bourg N, Brenguier L, Devaud C, Pasturaud P, Roudaut C et al (1995) Mutations in the proteolytic enzyme calpain 3 cause limb-girdle muscular dystrophy type 2A. Cell 81(1):27-40

Salim C, Boxberg YV, Alterio J, Fereol S, Nothias F (2009) The giant protein AHNAK involved in morphogenesis and laminin substrate adhesion of myelinating Schwann cells. Glia 57(5): 535-549

Spuler S, Carl M, Zabojszcza J, Straub V, Bushby K, Moore SA, Bahring S, Wenzel K, Vinkemeier U, Rocken C (2008) Dysferlin-deficient muscular dystrophy features amyloidosis. Ann Neurol 63(3):323-328

Uozumi N, Gao C, Yoshioka T, Nakano M, Moriwaki K, Nakagawa T, Masuda T, Tanabe M, Miyoshi E (2010) Identification of a novel type of CA19-9 carrier in human bile and sera of cancer patients: an implication of the involvement in nonsecretory exocytosis. J Proteome Res 9(12):6345-6353. doi:10.1021/pr100600u

von Boxberg Y, Salim C, Soares S, Baloui H, Alterio J, RavailleVeron M, Nothias F (2006) Spinal cord injury-induced upregulation of AHNAK, expressed in cells delineating cystic cavities, and associated with neoangiogenesis. Eur $\mathbf{J}$ Neurosci 24(4):1031-1041

Wenzel K, Carl M, Perrot A, Zabojszcza J, Assadi M, Ebeling M, Geier C, Robinson PN, Kress W, Osterziel KJ, Spuler S (2006) Novel sequence variants in dysferlin-deficient muscular dystrophy leading to mRNA decay and possible C2-domain misfolding. Hum Mutat 27(6):599-600 